\title{
Chain Extension and Thermal Behavior of Recycled Poly(Ethylene Terephthalate) Modified by Reactive Extrusion with Triphenyl Phosphite
}

\author{
Dan QIN ${ }^{1}$ a , Chaosheng WANG ${ }^{\mathrm{b}}$, Huaping $\mathrm{WANG}^{\mathrm{c}}$ and Xiaoting JIANG ${ }^{\mathrm{d}}$ \\ State Key Laboratory for Modification of Chemical Fibers and Polymer Materials, College of \\ Materials Science and Engineering, Donghua University, Shanghai 201620, China \\ atiffiny_qin@163.com, ${ }^{b}$ cswang@dhu.edu.cn, ${ }^{\text {c wanghp@dhu.edu.cn, }{ }^{\mathrm{d}} 1376413172 @ \text { qq.com }}$
}

\begin{abstract}
Reactive extrusion experiments of recycled PET fabrics (R-PET) were carried out in a Haake torque rheometer with triphenyl phosphite (TPP) and thermal behavior of modified R-PET was investigated by differential scanning calorimetry (DSC). The reaction mechanism which TPP acts as a cross-linker is verified by the experiment of phosphorus elemental analysis. DSC results show the presence of reaction residues may not modify melting temperature $T_{m}$ and crystallization temperature $T_{c}$ is controlled by the combined effect of molecular weight and reaction residues.
\end{abstract}

\section{Introduction}

PET undergoes degradation reactions during recycling processes such as mechanical, thermal and oxidative degradation leading to the reduction in intrinsic viscosity and mechanical property which may limit the recycling of R-PET. Reactive extrusion process has proved to be an effective way to enhance the molecular weight and properties of R-PET with chain extenders like phosphites[1].The reaction mechanism of PET in the presence of TPP has been investigated by several authors. S. M. Aharoni et al. [2] suggested that the reactions proceeded in two steps and the phosphite acted only as a catalyst and the phosphorus atom was excluded from the polymer chain. While Jacques et al. $[3,4]$ concluded that TPP acted as a cross-linker and the phosphorus atom remained within the main chain. The proper reaction mechanism of R-PET in the presence of TPP was investigated in the paper by the phosphorus elemental analysis. The measurement of DSC was used to study the thermal behavior of modified R-PET.

\section{Experimental}

\subsection{Materials}

Recycled PET fabrics (R-PET) used in this study were made by friction hot forming

*Corresponding author: tiffiny_qin@163.com 
technique with the intrinsic viscosity of $0.631 \mathrm{dl} / \mathrm{g}$. Triphenyl phosphite (TPP) was purchased from Acros Organics (CAS: 101-02-0).

Reactive Extrusion

R-PET was dried at $120^{\circ} \mathrm{C}$ for $24 \mathrm{~h}$ to avoid hydrolysis before processing. Reactive extrusion experiments were carried out in a Haake torque rheometer. The effect of TPP concentration on the modification was investigated by setting the chamber temperature at $260^{\circ} \mathrm{C}$ with a constant rotor speed of 40rpm and by varying the amount of additive from $0 \%$ to $7 \mathrm{wt} \%$. Impurities of purified samples were extracted with acetone in a soxhlet for $48 \mathrm{~h}$.

Phosphorus Elemental Analysis

The tests were performed using a highly efficient ICPAES (Prodigy, LEEMAN Company) to measure the phosphorus content in samples.

Differential Scanning Calorimetry (DSC)

Differential scanning calorimetry measurements were conducted by a TA Q20 thermal analyzer system under $\mathrm{N}_{2}$ atmosphere. The samples were heated from 30 to $300^{\circ} \mathrm{C}$ at the rate of $10^{\circ} \mathrm{C} / \mathrm{min}$ and kept for $5 \mathrm{~min}$ to eliminate thermal history and then cooled to $30^{\circ} \mathrm{C}$ at $10^{\circ} \mathrm{C} / \mathrm{min}$. A second heating was performed with the same heating protocol. During the process, crystallization temperature $\left(T_{c}\right)$ and melting temperature $\left(T_{m}\right)$ were recorded.

\section{Results and Discussion}

\subsection{The Way of TPP Addition}

Two groups of experiments were carried out in order to explore the optimized way to add TPP using 4\%wt TPP as an example: adding TPP at 0 min (previously reacted with R-PET before feeding) and adding after 5 min of mixing. The choice of the time for TPP addition was in order to obtain an homogeneous melt [3]. The value of torque is an indirect measurement to evaluate the chain extension. From Fig.1 we can observe the torque value of adding TPP at $0 \mathrm{~min}$ is lower than adding TPP at $5 \mathrm{~min}$ clearly, indicating that a lower molecular weight sample is obtained. There exists a competition between chain extension and thermal degradation. In case of the sample of adding TPP at $5 \mathrm{~min}$, a sharp increase in torque is observed after adding TPP indicated by arrows. After reaching a maximum at the time between 5 to $10 \mathrm{~min}$, the torque starts to decrease. It is possible that the chain extension reactions may be overlapped by the thermal degradation. Compared with adding TPP at $5 \mathrm{~min}$, there is only a slow increase in torque value in the enlarged view of the sample previously processed with TPP. The intrinsic viscosity of samples is listed in Table 1, as expected, adding TPP at $5 \mathrm{~min}$ is a better way.

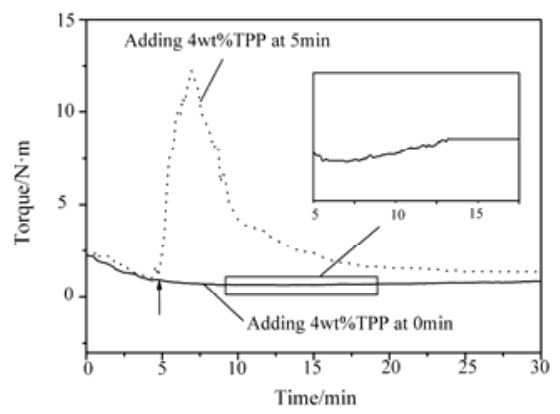

Fig. 1 Torque versus mixing time for R-PET with $4 \mathrm{wt} \% \mathrm{TPP}(\mathrm{TPP}$ was added at 5 min indicated by arrows) 
Table 1 Intrinsic Viscosity Of Samples

\begin{tabular}{cc}
\hline Ways of adding 4\%wt TPP & $\eta[\mathrm{d} 1 / \mathrm{g}]$ \\
\hline Adding TPP at $0 \mathrm{~min}$ & 0.857 \\
Adding TPP at $5 \mathrm{~min}$ & 1.077 \\
\hline
\end{tabular}

\subsection{Phosphorus Elemental Analysis}

Fig.2 presents the relationship between phosphorus content and mixing time during processing. The phosphorus content of purified samples increases with the mixing time which is in accord with Jacques's research results[3], indicating that the phosphorus is chemically bonded to the polymer chain. Chain extension reaction should be proceeded in the way shown in Fig.3 and Fig.4 [3,4]. As seen in Fig.3, the reactions between available hydroxyl group and phosphite could lead to triphosphite substitutions and chain extension. The proposed mechanisms between carboxyl chain ends and phosphite are given in Fig.4.

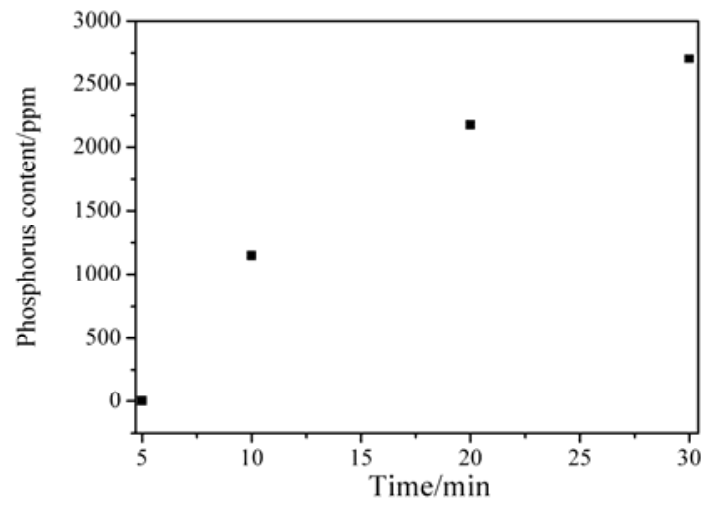

Fig.2 Phosphorus content versus time for R-PET with 5\%wt TPP after purification

\subsection{Effect of TPP Concentration on Intrinsic Viscosity}

The effect of TPP concentration on intrinsic viscosity of modified R-PET is shown in Fig.5, it is clear that intrinsic viscosity increases with the increment of TPP concentration at first, then it starts to decrease after reaching a maximum in the samples of $4 \% \mathrm{wt}$ TPP. The increase is an indication that chain extension reaction is taking place during reactive processing. The probable reason accounting for the decrease in intrinsic viscosity of the curve could be the chemical degradation caused by the side reactions resulting in polymer chain scission given in Fig.6 $[3,4]$. In the following sections of the paper, samples in the presence of $3 \mathrm{wt} \%, 4 \mathrm{wt} \%$ and $5 \mathrm{wt} \%$ TPP were used to investigate the thermal behavior of modified R-PET and T30, T40, T50 represent the corresponding samples for simplicity respectively. 


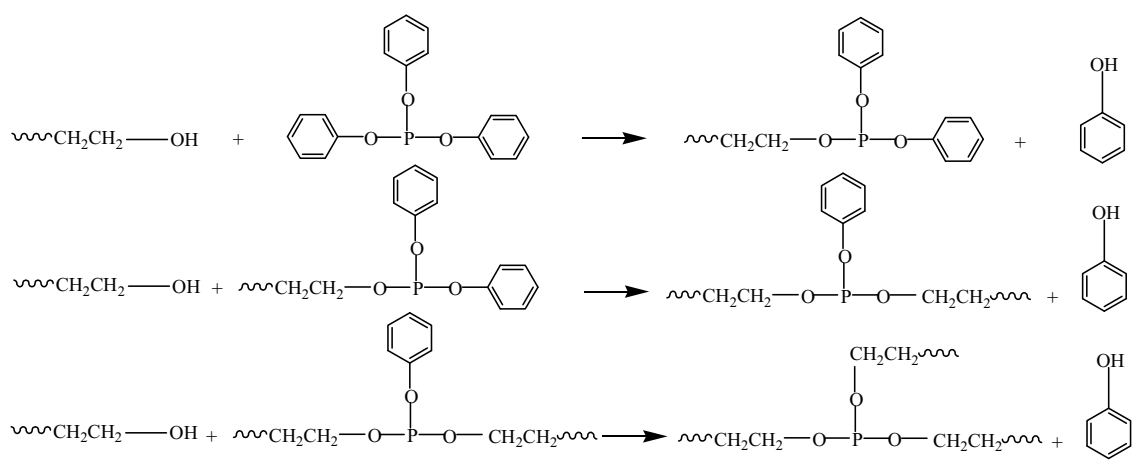

Fig.3 Reaction mechanism between available hydroxyl group and phosphite<smiles>[R]O[C@H](C(=O)O[Na])[C@@H](C)OP(O[R])O[R]</smiles><smiles></smiles>

Fig.4 Reaction mechanism between carboxyl chain end and phosphite

$\left(\mathrm{R}_{1}, \mathrm{R}_{2}=\right.$ phenyl group or polymer chain )

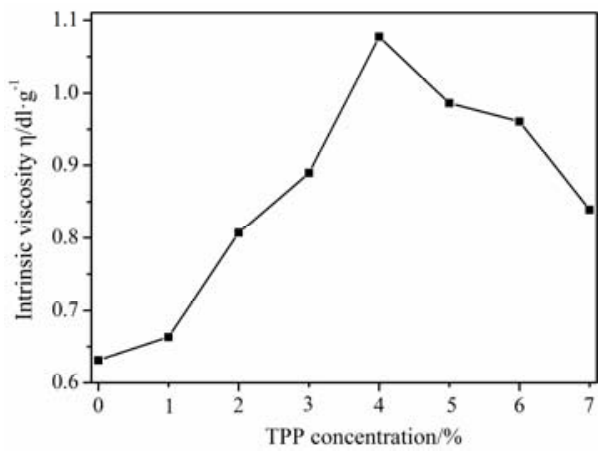

Fig.5 Effect of TPP concentration on the intrinsic viscosity of modified R-PET

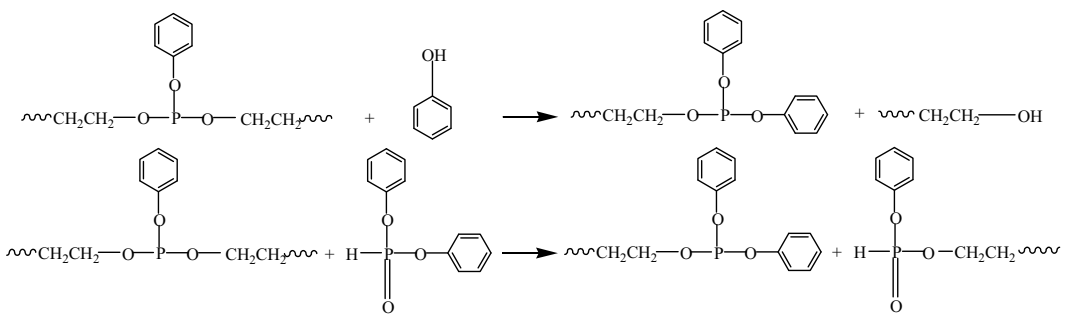

Fig.6 Side reactions resulting in polymer chain scission 


\subsection{Thermal Behavior}

In this work, samples were divided into two groups: one group were extracted with acetone in a soxhlet for $48 \mathrm{~h}$ to remove residues(purified samples) and the others with no treatment(unpurified samples). Temperatures of crystallization $T_{c}$ and melting $T_{m}$ are listed in Table 2. As for the unpurified samples, the increase of molecular weight and less chain flexibility caused by the chain extension reaction could lead to a slightly decrease of $T_{m}$ between untreated R-PET and modified R-PET. $T_{c}$ value of modified R-PET is increased in presence of TPP compared with R-PET. Dias et al.[5] claimed that reaction residues acted as the plasticizer in the unpurified samples to promote an easier crystallization. At the same time, samples with higher molecular weight could lead to the reduction of $T_{c}$. The relationship between the two effects seemed to be a competition.

$\mathrm{T}_{\mathrm{m}}$ value of purified and unpurified samples shares the similar variation tendency for different amounts of TPP. This fact indicates that the presence of reaction residues may not change the crystal perfection which is in accord with Dias's results[5].As mentioned previously, the crystallization is controlled by a pair of competitive reaction. Therefore only the effect of molecular weight could be observed in the purified samples, as a result, $\mathrm{T}_{\mathrm{c}}$ value of T50 is lower than that of T30 but higher than T40 between modified R-PET.

Table 2 Dsc Data Of R-Pet,T30,T40 And T50

\begin{tabular}{|c|c|c|c|c|c|}
\hline \multicolumn{2}{|c|}{ Items } & R-PET & T30 & T40 & T50 \\
\hline \multirow{2}{*}{$\mathrm{T}_{\mathrm{m}}\left[{ }^{\circ} \mathrm{C}\right]$} & Unpurified & 252.14 & 249.73 & 249.02 & 249.11 \\
\cline { 2 - 6 } & Purified & 252.14 & 248.63 & 247.76 & 247.78 \\
\hline \multirow{2}{*}{$\mathrm{T}_{\mathrm{c}}\left[{ }^{\circ} \mathrm{C}\right]$} & Unpurified & 197.38 & 198.15 & 198.68 & 201.47 \\
\cline { 2 - 6 } & Purified & 197.38 & 194.49 & 192.17 & 194.16 \\
\hline
\end{tabular}

\section{Conclusions}

The increase in phosphorus content of purified samples with the mixing time indicates that the phosphorus is chemically bonded to the polymer chain, which verifies the reaction mechanism TPP acts as a cross-linker. Thermal behavior of modified R-PET was studied by DSC and the results show that the increase of molecular weight and less chain flexibility caused by the chain extension reaction could lead to a slightly decrease of $T_{m}$ and the presence of reaction residues may not change the crystal perfection. $T_{c}$ value is controlled by the combined effect of molecular weight and reaction residues and for the purified samples extracted with acetone, only the effect of molecular weight could be observed.

\section{Acknowledgments}

This work was supported by the Fundamental Research Funds for the Central Universities (No. 2232013D3-17).

\section{References}

1. F.N. Cavalcanti, E.T. Teofilo, M.S. Rabello, S.M.L. Silva, Chain extension and degradation during reactive processing of PET in the presence of triphenyl phosphite, Polymer engineering and science. (2007)2155-2163. 
2. S. M. Aharoni, C. E. Forbes, W. B. Hammond, D. M.Hindenlang,F. mares, K. O'Brien, and R. D.Sedgwick, High-Temperature Reactions of Hydroxyl and Carboxyl PET Chain End Groups in the Presence of Aromatic Phosphite, Journal of polymer science: Part A: Polymer Chemistry. 24(1986) 1281-1296.

3. Jacques B, J. Devaux, R. Legras, and E. Nield, Reactions induced by triphenyl phosphite addition during melt mixing of PET/PBT blends: chromatographic evidence of a molecular weight increase due to the creation of bonds of two different natures, Polymer. 38(1997) 5367-5377.

4. B. Jacques,J. Devaux, and R. Legras, Investigation on model molecules of reactions induced by triphenyl phosphite addition during polyester processing, Macromolecules.29(1996)3129-3138.

5. M. L. Dias ,C. R. Nascimento, Thermal properties of post-consumer PET processed in presence of phosphites, Journal of thermal analysis and calorimetry.69 (2002) 551-559. 